\title{
Endoscopic treatment of leak at the tip of the "J" ileal pouch
}

\section{(이 $\odot \odot$}

Authors

Gursimran Singh Kochhar, Bo Shen

Institutions

The Interventional IBD Unit, Digestive Disease and Surgery Institute, The Cleveland Clinic Foundation, Cleveland, Ohio, United States

submitted 11.8.2016

accepted after revision $\quad 2.11 .2016$

\section{Bibliography}

DOI http://dx.doi.org/10.1055/s-0042-121664।

Endoscopy International Open 2017; 05: E64-E66

(c) Georg Thieme Verlag KG Stuttgart · New York

ISSN 2364-3722

Corresponding author

Bo Shen, MD, FASGE, The Interventional IBD (i-IBD) Unit, Digestive

Disease and Surgery Institute-A31, The Cleveland Clinic

Foundation, 9500 Euclid Avenue, Cleveland, $\mathrm{OH} 44195$, United

States

Fax: +1-216-444-6305

shenb@ccf.org

\begin{abstract}
Background and study aims The tip of the "J" of the ileal pouch is the vulnerable location for leak after restorative proctocolectomy, which has normally been treated with surgery. We aimed to describe a novel endoscopic method to treat the same.

Patients and methods A cohort of 12 consecutive patients with a leak at the tip of the "J" was identified in our prospectively maintained Pouch Registry. The endoscopic over-the-scope clipping (OTSC) system was used for the closure of the leak.

Results Eight patients (66.6\%) achieved complete closure of the leak documented by endoscopy confirmed with guidewire and/or contrasted pouchogram, with 6 requiring a single endoscopic session and 2 undergoing a repeat session. Four patients (33.3\%) had a persistent leak and required surgical intervention, of whom 1 developed abscess in the pre-spine region 14 days after the endoscopic procedure and underwent pouch revision surgery. Conclusions OSTC appears to be safe and effective in treating the leak at the tip of the "J" in the majority of patients.
\end{abstract}

\section{Introduction}

Ileal pouch-anal anastomosis (IPAA) with the "J" configuration after total proctocolectomy has become the standard surgical procedure for patients with ulcerative colitis (UC) or familial adenomatous polyposis who require colectomy [1]. The tip of the "]" consists of the distal ileum which is sealed with a linear stapler with or without additional reinforcement sutures. The tip of the "J" is 1 of the 2 most common locations for leaks, with the other being the anastomosis [2]. Our previous study of 27 patients with the leak at the tip of the "J" showed that salvage surgery was required in up to $93 \%$ of the patients with pouch repair, new pouch creation, or redo anastomosis [3].

We described the first case of endoscopic treatment of the leak at the tip of the "J" with the over-the-scope clipping (OTSC, the "Bear Claw") system in the literature [4]. Since then, the endoscopic therapy has become the first-line approach for the lesion in our Pouch Center/i-IBD Center. This case series is the natural extension of our previous case report.

\section{Patients and methods}

This cohort study was approved by our Institutional Review Board. The patients were identified from our prospectively maintained Pouch Registry. In addition, electronic medical records were reviewed and data were collected.

\section{Inclusion and exclusion criteria}

The inclusion criteria were patients with: 1) IPAA; and 2) a confirmed leak at the tip of the "J" on imaging and/or endoscopy. Exclusion criteria were patients with Crohn's disease-related fistula.

\section{Endoscopic procedure}

A leak from the tip of the "J" was confirmed either with a soft guidewire via pouchoscopy with or without pre- or post- endoscopy gastrograffin pouchogram ( $\triangleright$ Fig. 1 ). Risks and benefits of the endoscopic therapy were discussed with the patients and informed consent was obtained. The procedure was performed at our outpatient Interventional IBD Unit by a single experienced endoscopist (B.S.), without the need for fluoroscopic guidance.

Under conscious sedation and carbon dioxide insufflation, a GIF series gastroscope was used (Olympus, Tokyo, Japan). The contained leak was cleaned with hydrogen peroxide, betadine, and/or $50 \%$ dextrose. Endoscopic cytology brush was used to debride the epithelialized orifice of the cavity. Then 12-6tsized OTSC and 165-cm anchor (Ovesco Endoscopy USA, Cary, NC) were used to close the leak ( $\triangleright$ Fig. 2). 


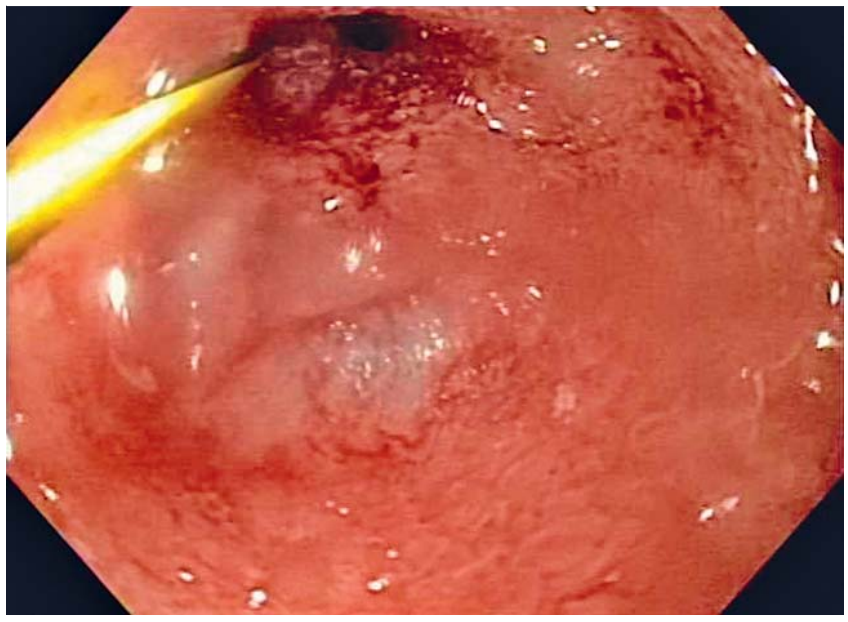

- Fig. 1 A leak at the tip of the "J" detected by soft-tip guidewire.

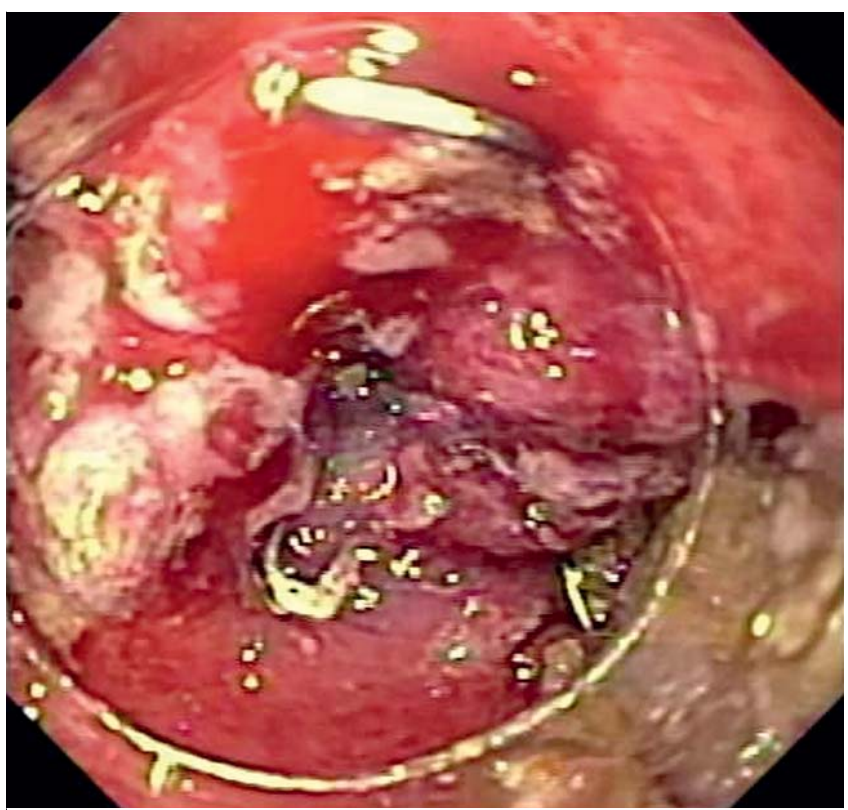

- Fig. 2 Successful closure of leaking from the tip of the "J" with the over-the-scope-clipping system.

\section{Outcome measurements}

The primary outcome was closure of the leak confirmed either by pouchoscopy with guidewire and/or gastrograffin pouchogram. Secondary outcomes were the need for surgical intervention and procedure-associated complications. Descriptive statistics were computed.

\section{Results}

\section{Demographic and clinical data}

A total of 12 patients were included. The mean age was $42.8 \pm$ 18.0 years with $5(41.6 \%)$ being male. All $12(100 \%)$ patients were Caucasians with average body mass index (BMI) of $24.0 \pm$ $3.0 \mathrm{~kg} / \mathrm{m}^{2}$ ( $\vee$ Table 1$)$. Of the 12 patients, $11(91.6 \%$ ) had a preoperative diagnosis of UC and 1 had indeterminate colitis. Diar- rhea was the main presenting complaint in 11 patients (91.6\%) followed by abdominal pain in 5 (41.6\%), and fever and leukocytosis in $1(8.3 \%)$. All 12 patients had chronic leaks, with a mean time from the pouch construction to diagnosis of the leak being $7.5 \pm 4.9$ years.

\section{Outcomes}

All 12 patients had successful deployment of OTSC during endoscopy. No excessive bleeding or perforation was observed. Ten patients $(83.3 \%)$ had a repeat pouchoscopy within 1 year after the index therapeutic pouchoscopy; 2 patients were followed up as outpatients with no additional pouchoscopy. Six patients $(50.0 \%)$ had complete healing on initial follow-up, 5 $(41.6 \%)$ had a recurrent leak identified on subsequent pouchoscopy and $1(8.3 \%)$ developed a new leak at different site other than prior treatment site. The latter 6 patients underwent repeat endoscopic therapy, 5 patients had reapplication of OTSC and one patient was attempted with endostitch after failed OTSC. Of these, 2 more patients achieved complete healing of the leak, while 4 (33.3\%) patients had a persistent leak requiring surgery.

There were no immediate post-procedure complications and all patients were discharged home the same day after routine post-procedural observation. One (8.3\%) patient, however, developed a pre-sacral spinal abscess 14 days after deployment of OTSC. That patient was admitted to the hospital and underwent a surgery for drainage and later on elective pouch revision and was treated with long-term intravenous antibiotics.

\section{Discussion}

Surgery-related complications are common in IPAA, with leaking at the tip of the "J" being one of the commonest. Risk of pouch failure is high when a septic complication occurs in the tip of the "J" [5]. The diagnosis may only be made at the time of salvage surgery in some patients and symptomatology such as abdominal pain, fever or diarrhea, is not specific [3]. Patients with the leak at the tip of the "J" may develop enterocutaneous fistula. Pouch endoscopy, contrasted pouchogram, and computed tomography or magnetic resonance imaging of the pelvis are common diagnostic modalities. To date, management of leaking at the tip of the "J" has been exclusively surgical, including procedures like repair and redo pouch $[6,7]$. This case series describes a novel endoscopic management of this pouch complication that may obviate surgical intervention by minimizing cost and avoiding risks associated with surgical procedures.

This is the first case series in the literature showing successful use of the OTSC system for management of leaking at the tip of the "J". Of the12 patients in the series, 8 (66.6\%) were successfully treated with OTSC. Our series shows that use of OTSC for leaking at the tip of the "J" is a valid alternative. Besides being a technically feasible procedure, OTSC appears to also be safe.

OTSC has gained popularity among the endoscopy community. It has been used to successfully treatd non-variceal gastrointestinal bleeding [8], fistula of the gastrointestinal tract [9], and esophageal perforation [10]. In a recent large single-center 
- Table 1 Clinical characteristics of patients treated with over-the-scope clip for the leak at the tip of the "J."

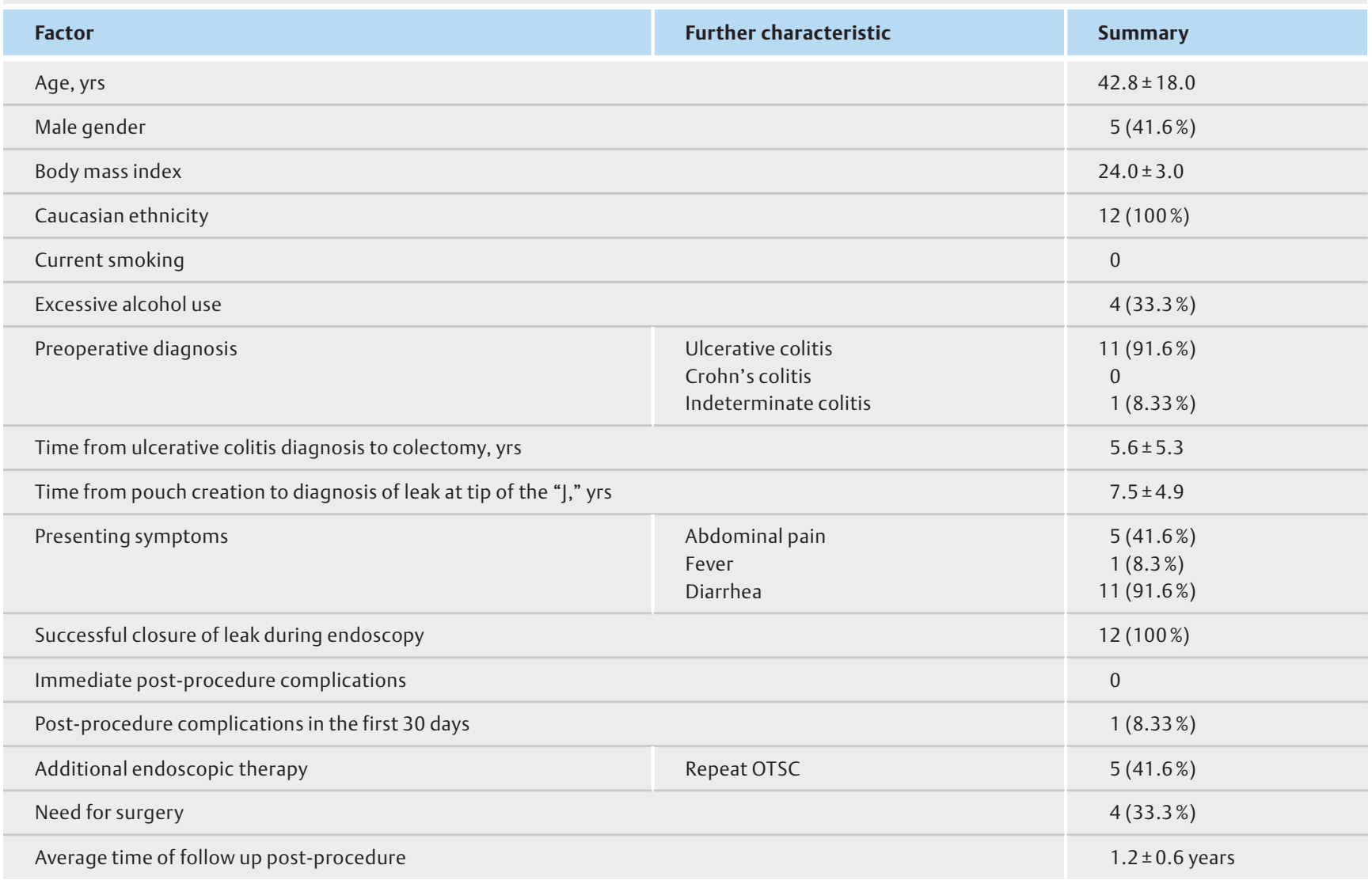

case series, OTSC was used to treat upper and lower gastrointestinal bleeding, gastrointestinal perforation, fistula, and perforation in 84 patients with a success rate of $92 \%$, with no reported complications [8].

Our study has few limitations. Being a historical cohort case series, it has inherent bias. It is also a single-center, tertiarycare based, non-controlled study, which may limit the generalizability of the results to the general IBD population. It is, however, difficult to perform a randomized controlled study in this patient population.

\section{Conclusion}

In conclusion, leaking from the tip of the "J" in patients with IPAA can be effectively and safely treated with over-the-scope clipping system.

Competing interests

None
References

[1] Fazio VW, Kiran RP, Remzi FH et al. Ileal pouch anal anastomosis: analysis of outcome and quality of life in 3707 patients. Ann Surg 2013; 257: 679-685

[2] MacRae HM, McLeod RS, Cohen Z et al. Risk factors for pelvic pouch failure. Dis Colon Rectum 1997; 40: 257-262

[3] Kirat HT, Kiran RP, Oncel M et al. Management of leak from the tip of the "J" in ileal pouch-anal anastomosis. Dis Colon Rectum 2011; 54: $454-459$

[4] Lian L, Shen B. Closure of leak at the tip of the "]" after ileal pouch-anal anastomosis using a novel over-the-scope clipping system. J Coloproctol 2014; 34: 120 - 123

[5] Heuschen UA, Allemeyer EH, Hinz U et al. Outcome after septic complications in J pouch procedures. Br J Surg 2002; 89: 194-200

[6] Pellino G, Selvaggi F. Outcome of salvage surgery for ileal pouch complication and dysfunction. The experience of a referral Centre and review of literature. J Crohns Colitis 2015; 9: 548-557

[7] Remzi FH, Fazio VW, Kirat $\mathrm{H}$ et al. Repeat pouch surgery by the abdominal approach safely salvages failed ileal pelvic pouch. Dis Colon Rectum 2009; 52: 198 - 204

[8] Wedi E, Gonzalez S, Menke D et al. One hundred and one over-thescope-clip applications for severe gastrointestinal bleeding, leaks and fistulas. World J Gastroenterol 2016; 22: $1844-1853$

[9] Goenka MK, Goenka U. Endotherapy of leaks and fistula. World J of Gastrointes Endosc 2015; 7: 702 - 713

[10] Sanaka MR, Raja S, Thota PN. Esophageal perforation after pneumatic dilation for achalasia: successful closure with an Over-the-Scope clip. J Clinical Gastroenterol 2016; 50: 267-268 